\title{
Direct Imaging of Core-Shell Structure in Ag-Au Nanoparticles
}

\author{
Z.Y. Li, * J. Yuan, ** Y. Chen, * R.E. Palmer* and J.P. Wilcoxon*** \\ * Nanoscale Physics Research Laboratory, School of Physics and Astronomy, The University of \\ Birmingham, Birmingham B15 2TT, U.K. \\ ** Department of Material Science and Engineering, Tsinghua University, Beijing, China. \\ *** Nanostructures and Advanced Materials Chemistry 1122, Sandia National Laboratory, \\ Albuqurque, N.M. 87185-1421, U.S.A.
}

Bimetallic nanoparticles have been attracting great interests recently due to their potential applications in technologies such as catalysis, electronics and optical devices. The chemical and physical properties of bimetallic particles can be tuned not only by varying their size, but also their composition. At present, it is still a significant challenge to characterise the internal structures of bimetallic nanoparticles. Optical methods are the most commonly used approach for bimetallic nanoparticle analysis. However, for core-shell bimetallic particles this method has to be used with caution, because (1) the optical contributions from the core material may be effectively screened, and (2) for sufficiently small particles, the damping of the plasmon at the particle-medium interface will dominate the dispersion. In any case, optical measurements provide average information that may not reflect the heterogeneous nature of some bimetallic nanoparticle systems. In this paper, we present an alternative method of nanostructural determination using the high angle angular dark field (HAADF) imaging technique in electron microscopy.

We studied two types of materials, pure Au particles and bimetallic Ag/Au particles with core/shell structure. The nanoparticles were prepared using the inverse micelle method. They were synthesized by first growing an $\mathrm{Ag}$ seed nanocrystal of $3 \mathrm{~nm}$ diameter followed by several generations of $\mathrm{Au}$ overlayers. The particles were passivated with a $\mathrm{C}_{12} \mathrm{H}_{25} \mathrm{~S}$ alkanethiol monolayer and dissolved in toluene. A drop of the solution was deposited onto a TEM copper grid covered with an amorphous carbon film in ambient conditions. The samples were investigated using a Tecnai TEM system.

Fig. 1 shows HAADF images of three samples of different nanoparticles, all obtained under identical incoherent imaging conditions. Using the homometallic nanoparticles in (b) as a reference, we can see that the two bimetallic nanoparticle systems show distinctly different internal contrast variation. The image in (a) exhibits nearly flat contrast at the core, while the image in (c) has a more intense contrast at the centre. The effect is more clearly seen in line profiles (Fig.2).

It is known that the intensity in HAADF images is proportional to the power of 1.7 of the average atomic number $(Z)$ of the atomic column. The atomic numbers of $\mathrm{Ag}(47)$ and $\mathrm{Au}(79)$ are sufficiently different so that the Z-contrast imaging method should be capable of determining the possible composition modulation within the nanoparticles. This is validated through a simulation of the core-shell structure based on simple geometrical considerations, in which the particles are assumed to have spherically symmetrical structures, and the HAADF intensities are taken to depend exclusively on the height of the projected atomic column. The simulation results are shown in Fig. 3 for five kinds of particles with different internal structures: a pure Au nanoparticle, two Ag core/Au shell nanoparticles and two $\mathrm{Au}$ core/Ag shell nanoparticles. The atomic ratio of the $\mathrm{Ag}$ and $\mathrm{Au}$ compositions has been chosen as 1:2 or 2:1. It is clear that the Z-contrast line profiles for the different particle structures have different shapes. For a homometallic particle, a smooth hill shape is 
seen. For $A g_{\text {core }}-\mathrm{Au}_{\text {shell }}$ particles, a dimple appears at the centre of the profile as expected given the lower $\mathrm{Z}$ number of $\mathrm{Ag}$ than $\mathrm{Au}$. When the $\mathrm{Ag}$ : $\mathrm{Au}$ composition ratio changes from 1:2 to 2:1, the overall shape of the profile remains similar. However, for particles with an inverted internal structure, i.e., Au core and Ag shell, the dimple changes to a pointed hill shape.

The similarity in experimental and simulated HAADF contrast variation of the particles with $A g_{\text {core }}: \mathrm{Au}_{\text {shell }}=1: 2$ (the $\mathrm{Au}$ shell is thick) confirms the existence and the long-term stability of the core-shell structure, in accordance with the metal reduction sequence. On the other hand, the unexpected result revealed in Fig.1(c) for the nominally $\mathrm{Ag}_{\text {core: }} \mathrm{Au}_{\text {shell }}=2: 1$ (the $\mathrm{Au}$ shell is thin) can only be understood if the initial Au shell structure growing on the Ag seed crystal is unstable against Ag replacement. This could be the result of ageing, since optical measurements of the fresh samples showed the cluster absorption peak red-shifts monotonically with increasing amounts of $\mathrm{Au}$, consistent with the formation of a core/shell structure. The proposed diffusion of the Ag atoms from the core over time may be attributed to the thermodynamics of the process.

In summary, we have demonstrated that the HAADF imaging is a powerful technique to study the structure as well as the evolution of core-shell nanoparticles down to size of $\sim 4 \mathrm{~nm}$. From the HAADF images and with the aid of a simple geometric simulation, we have further demonstrated that nanoparticles produced by sequential growth of gold overlayers on Ag seeds do indeed possess core-shell structure, though the Ag/Au interface may be blurred by atomic inter-diffusion. The kinetics of achieving core-shell structures requires further investigation.

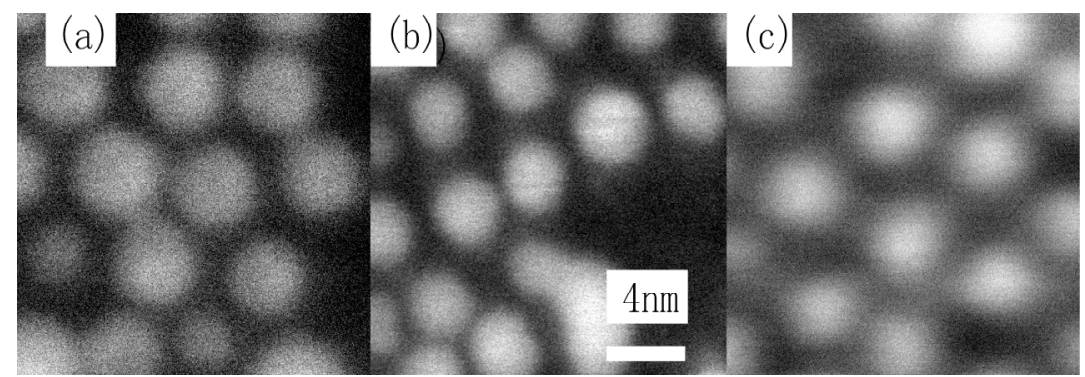

FIG.1 HAADF images from nanoparticles, (a) $\mathrm{Ag}_{\text {core }}: \mathrm{Au}_{\text {shell }}=1: 2$; (b) $\mathrm{Au}$; and (c) $\mathrm{Ag}_{\text {core }}: \mathrm{Au}_{\text {shell }}=2: 1$.
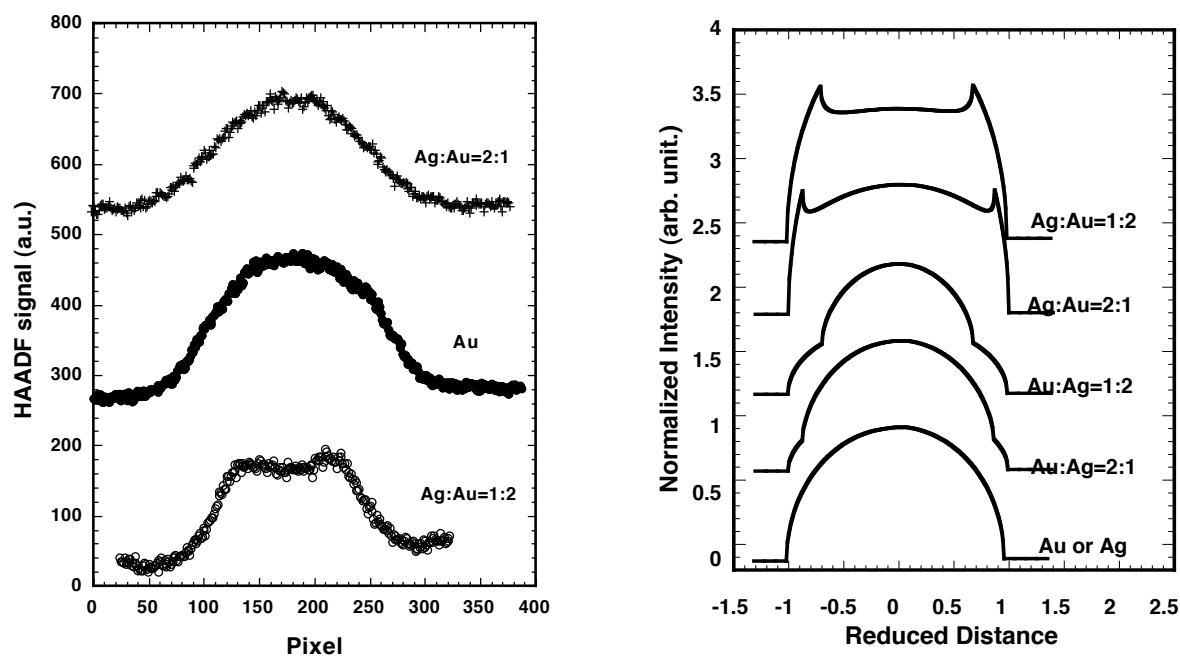

FIG. 2 (left) Typical line-profiles obtained from particles in the images in figure 1.

FiG. 3 (right) Simulated Z-contrast line-profiles across spherical nanoparticles. 